\title{
EUNIS 2013: Cloud Service Delivery Across the R\&E Community - Opportunities and Risks
}

\author{
Karl Meyer ${ }^{1}$, Panos Louridas ${ }^{2}$, Andres Steijaert ${ }^{3}$, Fulvio Galeazzi ${ }^{4}$, Miroslav Ruda ${ }^{5}$, Branko Radojevic ${ }^{6}$ \\ ${ }_{1}^{1}$ DANTE Ltd, City House 126-130 Hills Road, Cambridge, CB2 1PQ, UK, karl.meyer@dante.net \\ ${ }^{2}$ GRNET, 6, Mesogion Av. 11527, Athens, Greece, louridas@grnet.gr \\ ${ }^{3}$ SURFnet, P.O. Box 19035, 3501 DA Utrecht, The Netherlands, andres@surfnet.nl \\ ${ }^{4}$ GARR, Via dei Tizii, 6, 00185 Roma, fulvio.galeazzi@garr.it \\ ${ }^{5}$ CESNET z. s. p. o.,Zikova 4,160 00 Praha 6, Czech Republic, miroslav.ruda@cesnet.cz \\ ${ }^{6}$ CARNET, Josipa Marohnića 5, 10000 Zagreb, Croatia, branko.radojevic@carnet.hr
}

\section{Keywords}

Cloud Services, Standards, Data Integrity, Brokerage, Best Practice, Research and Education.

\section{Copyright notice}

This document has been produced with the financial assistance of the European Union. The contents of this document are the sole responsibility of DANTE and can under no circumstances be regarded as reflecting the position of the European Union. Copyright 2009-2013 DANTE Ltd. All rights reserved.

DANTE is the trading name of:

Delivery of Advanced Network Technology to Europe Limited

Company registration number: 2806796

Registered in England

\section{ABSTRACT}

Cloud computing, and cloud services in particular, offer the Research and Education sector huge opportunities to both maximise effectiveness and reduce the capital investment and development time to deliver results. By utilising shared and off-the-shelf services for commodity activities, the R\&E community can refocus its design, development and support resources into those fields that cannot be easily provided by the commercial sector.

Cloud computing empowers users to select and use the services they really want, in an easy and often economically attractive manner. The broad standardisation of service delivery offers substantial advantages with scalability and user acceptance. By using services that the users have had experience of outside the R\&E community, training requirements can be minimised and personal efficiency can be improved.

The scalability of cloud services also allows rapid expansion or contraction of capacity as the project requires with minimal penalties. This near-linear cost model allows easier budgeting and financial control.

\subsection{Cloud Services - Removing Barriers and Borders}

R\&E is increasingly an international environment with collaboration between teams and across borders being the norm within many fields. IP networking has helped bridge borders and removes distance from the R\&E equation. Currently there are over $800 \mathrm{EC}$ funded research projects with collaborators outside the EU, IP networking and cloud services offer the facility for enabling and enhancing these activities by allowing near universal access to systems and applications.

High Capacity networking has turned the R\&E community into a truly global village and cloud services will aid this collaboration even further. By moving services and applications from the edge of the network into its center, access to these services is no longer constrained by capacity and 
availability at the edge of the network. This has huge implications and opportunities for university and research campus networks and services and, of course, the research and faculty staff that rely on them and the IT teams that deliver and support them. This paper examines the taxonomy of Cloud Computing and Cloud Services and identifies the opportunities lying within each genre of service offering and how R\&E organisations can most benefit from them.

\subsection{Risks and Threats from Cloud Services}

Alongside these opportunities there are also a range of risks that need to be recognised and addressed.

The standard delivery of these services by commercial (and often US based) organisations can often be incompatible with the requirements of higher education and research.

There are significant challenges on trust, security, privacy, legislation and regulation. These issues have different implications between cloud services used in a private capacity compared to services used within a research environment where the ownership of data and the need to ensure strong custodial control are paramount.

There are also issues regarding data portability and interoperability. Commercial service providers have a commercial imperative to maintain users and reduce churn within their user base and so have little incentive to collaborate with competitors on these issues.

These are cross-border phenomena which have a major impact on the research and education community. It is therefore essential that higher education and research collaborate on a European level, so that the benefits of the cloud can be fully realised and the attendant risks are fully understood and appropriately managed.

By presenting a united front, the R\&E community, from network managers to research project managers, and from students to faculty, can work to guide and influence cloud service providers in these areas.

This paper will highlight how GÉANT is working to mitigate these risks through a coordinated approach and implementation of a range of Best Practices across the community. In addition, through developing a range of procurement guidelines, collaboration can reduce the learning curve for brokering these services and minimise duplication of standards and policies.

GÉANT is the pan-European research and education network that interconnects Europe's National Research and Education Networks (NRENs); together connecting over 50 million users at 10,000 institutions across Europe, supporting research in areas such as energy, the environment, space and medicine.

\section{BACKGROUND AND REASONING}

Cloud computing is rapidly changing the way users obtain and utilise IT services. The cloud empowers users to select and use the services they really want, in an easy and often economically attractive manner. The delivery of services from the cloud has assumed a substantial momentum in higher education and research, and is a pressing topic. There are challenges with regard to trust, security, privacy, legislation and regulation, at national and European level. There are also issues regarding data portability and interoperability. In their "standard appearance", cloud services are often incompatible with the requirements of higher education and research, which prevent higher education and research institutes from embracing the cloud.

The cloud is a cross-border phenomenon that has a major impact on IT. As a vendor neutral, European ICT project, GÉANT can play a major role at both a strategic level and delivering end user services. Through GÉANT's service development in the cloud arena, European NRENs will collaborate to overcome the challenges mentioned above and will enable and facilitate higher education and research to adopt the cloud on a large scale, with the right conditions of use. 
The effort has strong links with other services provided by GÉANT, and is targeted to exploit other cross-border network services developed by the NREN/GÉANT community for the benefit of the cloud environment.

Effective Cloud Services are predicated on ubiquitous access to services. Therefore the issue of effective mobile data service is closely linked to the GEANT cloud activity, which the project acknowledges the growing importance of mobility to researchers' work. The high costs associated with roaming and decoupling from the standard AAl still make nomadic work difficult. The objective is to analyse and eventually provide a service that suits researchers' needs. This activity marks the start of NRENs' cooperation on cloud initiatives that will lay the groundwork for future activities within the EU Horizon2020 program.

\subsection{Taxonomy of Cloud Services}

The cloud service environment is a complex and diverse one with a number of separate and unique offerings. It ranges across a spectrum of applications and user types. This environment can be described by reference to a taxonomy of services each with a different profile and set of requirements. These can be described in the following manner

\section{- Cloud Computing}

- Grid Computing - high performance, distributed processing clusters combined together for special purpose activities. One user to many machines.

- Cloud Servers - usually VM based virtualised processing with many users to one physical machine. Many cloud services will operate in a many to many environment with each physical server divided into VMs then users acquiring multiple VMs and operating a distributed, resilient model. The applications tend to be more "commodity" web/database applications.

- Cloud Based Special Purpose Services - Provided on a one service/server farm to one client basis. Usually operated as a virtualised data centre solution. More akin to a traditional outsourcing contract. Applications will tend to be bespoke or highly customised variants of commercial software. Usually allied with a VPN or private networking solution. This model is usually restricted to very large and/or multi-national organisations with specialist outsourcing companies offering the services.

- Cloud Business Services - Typically used for organisation wide, corporate services (for example SAP or Salesforce). Formatted in a many users to one service environment using public networking infrastructure and secured by an AAI and SSL system. Within the R\&E community applications such as finance, payroll and HR could be offered through this model.

- Public Cloud Services - The most commonly used and understood cloud services, typified by services such as Dropbox, Hotmail, etc. These services usually have somewhat basic AAl and/or security. Many business users will find staff using these services for business data and activities.

Within this model there are many sub variants and hybrids. It is within the fields of Cloud Business Services and Public Cloud Services that the greatest opportunities arise for the GÉANT Support to Clouds project to assist buyers, commissioning bodies and users select and use the correct service model for their needs.

\subsection{Objectives}

The objectives of the GEANT Cloud services group is to ensure that the combined network, middleware and cloud service, offered by GÉANT and NRENs, stands as a key technical and financial element in the ecosystem, and delivers to the users and campus network just what is needed.

- Recommend a cloud strategy, based on shared principles and values.

- Part of the work will be to build on the existing cloud service initiatives of the GÉANT partners (NRENs) to quickly bootstrap services for the broader GÉANT community (NRENs, university and research campus, research projects, etc.). 
- Develop policies, requirements and rules to establish standards and interoperability, on issues such as trustworthiness, security and business models.

- Aggregate demand and negotiate with cloud providers, both commercially and within the GN3plus community; acting as a broker between the service providers and users to establish the right conditions of use. Where possible, leverage existing brokerage agreements of partner NRENs.

- Exploiting advanced AAI middleware and network infrastructure and investigation of the operation of a mobile data service, closely aligned or integrated with the GÉANT eduroam service.

- Establish knowledge dissemination and information exchange within the community on cloud computing, in close collaboration with the TERENA Task Force.

- Ensure that the combined network, middleware and cloud service, offered by GÉANT and NRENs, stands as a key technical and financial element in the ecosystem.

- Build experience and provide a better understanding of the role of GÉANT in the cloud computing landscape, offering a strategy for future work under Horizon2020.

The following are five focus areas are at the core of the GEANT cloud services:

- Cloud Strategy.

- Cloud Standards and Interoperability.

- Cloud Brokerage and Vendor Management.

- Cloud Integration.

- Mobile Data Service.

\section{CLOUD STRATEGY}

\subsection{Objectives}

The needs of end users (demand side) and the capabilities of service providers (supply side) result in a compelling, but complex, landscape of opportunities and challenges. The primary goal is to identify and describe the needs of the community, relate these to the capabilities of the market and community, and recommend a cloud strategy, which can be used by GEANT and NRENs. The cloud strategy centers around networking and interconnecting the cloud capabilities of the community and commercial providers, building on the role of the NRENs. It forms the foundation for the adoption of the cloud by research and education, via the advanced network capabilities from NRENs. It also presents overall guidelines for the other focus areas.

\subsection{Work Plan}

This part of the project will develop and manage a cloud strategy and principles. It contains a description of:

- Community / user needs and supplier capabilities.

- Overall goals and principles regarding the use of cloud computing and cloud services in higher education and research.

- Core competencies needed to organise and adopt the cloud (both from a consumption and production perspective).

- The added values, benefits and "unique selling points" (distinctive features and differentiating elements) GÉANT and NRENs can offer to higher education and research.

- More organisational and technical structures.

We will build on existing best practices, business cases and experiences in the community and other environments (e.g., commercial, not-for-profit, public environment and EU communications). 
The recommendations on strategy and principles will help GÉANT and NRENs define business cases for cloud services and establish a set of shared "cloud values". This alignment enables them to use a common language while collaborating with each other and when interacting with other organisations and stakeholders.

The aim is to establish interactions on a strategic level with other cloud initiatives within research and education and relevant entities outside of the community. Special attention will be paid to understanding how the cloud paradigm can serve research objectives and will look at the potential opportunities from the creation of a European e-infrastructure cloud initiative forum. In addition to GÉANT, this initiative may encompass players such as EGI, EUDAT, CLARIN and Digital Libraries, and can contain cooperation with Helix Nebula.

\section{CLOUD STANDARDS AND INTEROPERABILITY}

\subsection{Objectives}

Cloud services vary greatly in the detail of the specification of their service offerings, even where these services may appear identical. Examples of differences include: Service Level Agreements, security, privacy and data portability. This lack of consistency makes it difficult to compare different services and to select the appropriate service for a particular situation in the research and education context. This impedes the adoption of cloud services. The standardisation of cloud services, on a European and global level, will reduce the entry-barriers, increase interoperability and enable large-scale use.

This element of the project will:

- Investigate and describe the needs of the research and education community regarding interoperability of cloud services.

- Look at available standards that can provide this interoperability and can be reused and define gaps where current standards need to be improved or new standards need to be developed.

- Liaise and collaborate with relevant standardisation bodies, cloud and e-infrastructure providers (both commercial and community based) to help achieve commonly accepted standards, relevant for research and education.

- Promote the use of available standards (in the community and by service providers) and the development of standards that will greatly facilitate such integration. The collaboration between main e-infrastructure in Europe includes fostering standardisation as a key component.

\subsection{Work Plan}

The aim of this element of the project is to help GÉANT get involved and participate in the standardisation domain. It allows GÉANT to learn from others in this field and understand how it applies to GÉANT as an aggregator. This helps to define best practice and recommendations applicable to both the NREN community and vendors.

Some of the specific standardisation areas will include:

- Polices and rules to enable interoperability (including data portability) between providers, in order to avoid vendor lock-in.

- Promoting a standardised set of specifications and best practices for service parameters accepted by providers and the higher education and research community.

- Expressing the needs of the GÉANT user community regarding European principles on data security and privacy.

- Collaborating with other European e-infrastructure providers that provide/facilitate community cloud services in order to enable seamless delivery of cloud services to NREN users. 
This will produce a long-term view of standardisation, coordination and mediation between policy, legal and technology domain expertise.

We will represent GÉANT within European cloud initiatives (currently driven by the EC) to address the standardisation aspects. We will seek to develop a leadership role based on GÉANT's domain expertise and raw aggregated purchasing power. We will also closely collaborate with other einfrastructure providers to develop Memoranda of Understanding, interconnection policies and technical methods to facilitate the best possible integration of the service with GÉANT.

The core activities of this element of research will be to:

- Collaborate with other elements of the project to identify solutions that could be generally applicable and standardised.

- Collaborate with external stakeholders, such as other e-infrastructures, standards development organisations, service providers and the EC, to develop mutually acceptable standards.

\section{CLOUD BROKERAGE AND VENDOR MANAGEMENT}

\subsection{Objectives}

The goal is to acquire and manage the delivery of services from providers to the pan-European GÉANT community. These parties can be commercial vendors as well as NRENs, and other research and education communities. This task strives for an attractive, well-balanced portfolio of cloud services.

The aim is not seek to replace existing NREN brokerage activities, but to act as a coordination mechanism to amplify these NREN brokerage activities to a pan-European scale.

Such coordination should help to increase the attractiveness of the terms offered by providers, by demonstrating an aggregated pan-European demand. It should also increase the efficiency for both providers and NRENs by reducing the number of negotiations and by making negotiated agreements (frameworks) available to the GÉANT community. This Task will fully engage with the existing NREN brokerages to promote an efficient and coordinated pan-European approach, where appropriate, by building on existing experience and supplier relationships.

By aggregating the tens of millions of GÉANT users, we will help to establish the world's largest single research and education community for cloud services. If mobilised effectively, the GÉANT Cloud Brokerage could develop into one of the most influential users of cloud-based services, and as such, play a significant role in the direction and development of cloud services, both within Europe and elsewhere.

\subsection{Work Plan}

The aim here is to gather the NRENs' national brokerage activities into a focused effort towards service providers, to secure agreements on behalf of the GÉANT community: to negotiate and secure the best possible terms, to enable NRENs and their customers to obtain access to these services.

Although the cloud is a fast-emerging technological force, its contractual and legal aspects are still in their infancy. A European approach and multi-year effort is needed to reach a more mature level. As a result we will therefore work towards establishing robust and long-term relationships with service providers.

\section{CLOUD INTEGRATION}

\subsection{Objectives}

Cloud services need to be connected to the IT infrastructure of the NREN community: the networks and AAl middleware. There is a need therefore to facilitate this technical integration and implementation work. 


\subsection{Work Plan}

The aim is to provide technical expertise for integrating cloud services with the GÉANT and NREN networks and AAI middleware, on a global IT architectural level as well as on technical details. The aim is to:

- Gather and produce best practices, create instructions and support material, and provide advice to the community on how integration can take place.

- Aggregate NRENs' plans to adopt a cloud services and align timelines and resources, to facilitate community collaboration and cooperation with vendors.

- To coordinate these joint-integration efforts and vendor contacts to assure the required vendor support.

- Take results from standardisation and brokering efforts, and integrate these with the capabilities of the NRENs.

- Collaborate closely with service providers to determine the technical requirements for connecting capable providers to the GÉANT and NREN infrastructures (network, AAI middleware).

- Work to ensure that the lessons learned during the implementation work can be applied to the relevant standardisation efforts.

- Liaise with other GÉANT Activities related to cloud integration technology.

- Work with service providers and the relevant Service and Joint Research Activities, define and liaise with other GÉANT/NREN service experts to provide solutions that match these requirements and ensure that they are appropriately delivered.

\section{MOBILE DATA SERVICE}

\subsection{Objectives}

Higher education and research users are rapidly adopting mobile devices and a "nomadic work style", both on and off campus. They want to be able to use online services anytime and anyplace. However, the high costs associated with roaming and decoupling from the standard AAI make mobile work difficult.

There is a huge demand to facilitate the user needs and remove these financial and technical obstacles. As a result, NRENs have to extend their network capabilities and role as network provider towards mobile data networks. The objective is to provide mobile data network capabilities to GÉANT and NRENs, which suit the needs of education and research.

The service needs to be closely aligned or integrated with the GÉANT eduroam ${ }^{\circledR}$ service, thus providing a consistent service presentation and user experience.

\subsection{Work Plan}

This part of the project will:

- Analyse community requirements (users, NRENs, GÉANT) and community and market capabilities based on information provided from the results on mobile data work in GEANT.

- Advise on the next steps: procurement, implementation and exploitation scenarios:

- Prepare for the operational stage of the mobile data service. 


\section{TASK INTEGRATION AND WORKFLOW}

The following diagram indicates the interactions and workflows between the various tasks within this activity

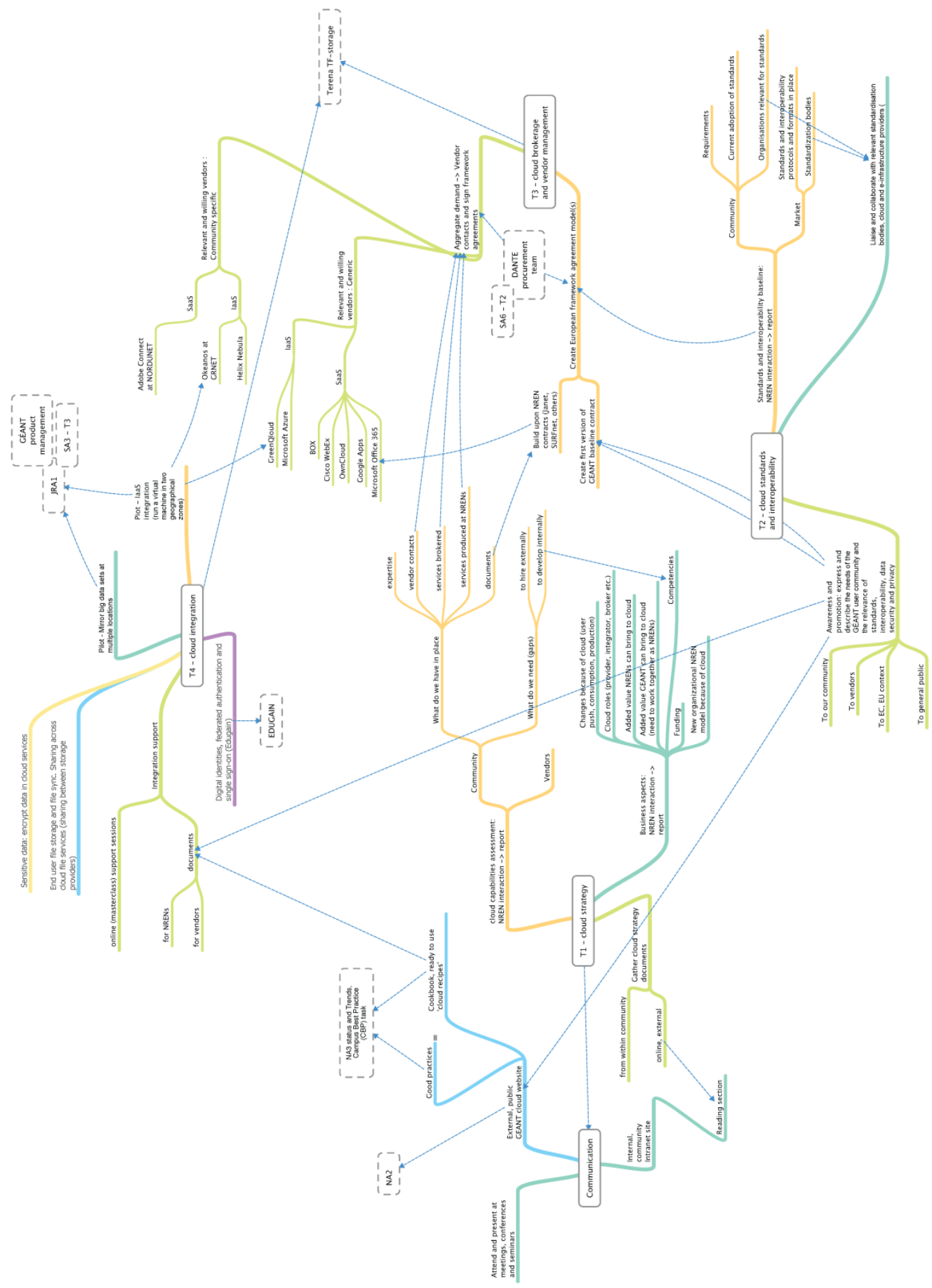

Figure 1. "Support to Clouds" Activity Workflow 


\section{CONCLUSION}

The opportunities offered by Cloud Services within the R\&E environment are potentially huge. They offer a paradigm shift of a similar, if not greater, magnitude than that created by the development of high performance networking.

Cloud Services offer the opportunity to provide ubiquitous access to shared services and data without reference to artificial boundaries of geography and organisation. They can offer a more scalable solution to the needs for expansion of services and can provide an alternative delivery mechanism to the previous capital intensive and complex management of locally distributed systems.

The ability to aggregate users and loading from multiple organisations within a combined delivery mechanism not only potentially reduces delivery costs but can also reduce both on-going support costs and the environmental costs of operating distributed systems.

The opportunity for effective Public Private Partnership is enhanced by the possibility of aggregating requirements, brokerage of services on a pan-European basis and simplifying contractual structures. There is also the opportunity for risk reduction as a result of contracting for known and understood services rather than custom development of bespoke solutions.

Countering these opportunities is a series of risks. Some of these are generic to Cloud Services and some are specific to the environment that the R\&E community creates.

The issues of data integrity, protection, transparency and cross-platform mobility are common across all cloud services and particularly so within the corporate and R\&E sectors where the data and services used can form an essential element within the organisations' activities. This activity will seek to address these issues with its range of tasks reflecting Contracting, Best Practice, Standards and Brokerage. This work will not only have relevance to the R\&E sector but, it is hoped, will help guide better Cloud Service provision across Europe and the World.

Within the R\&E sector there are a number of specific operational attributes that are unique (or potentially unique) to the environment. In particular, user security and identity across organisational boundaries are emerging issues that need to be addressed urgently. Indeed as the development of high speed R\&E networking has removed geographical boundaries, the issues relating to organisational boundaries can be seen to have been exacerbated. More and more researchers want and need access to services provided by other institutions. The provision of cloud services will only accelerate this requirement.

This "Support to Clouds" activity recognises this as a major bottleneck and so the task dedicated to this area (Cloud Integration) will be working closely with the other AAl and security activities within the GÉANT project to ensure the infrastructure is in place to support the growth and development of Cloud Services across the R\&E Community.

With this multi-task and multi-track approach this "Support to Clouds" activity is well placed to help ensure the development of Cloud Services in the R\&E community meet the needs of the Service Providers, R\&E institutions and, most importantly, the end users.

GÉANT - with its long history in the provision of multi-national, multi-domain networking and infrastructure services to the R\&E community - has proven experience in the field of inter-NREN cooperation and the development of open accessible standards within IT services. This places GÉANT at the forefront of the work in this emergent field.

\section{REFERENCES}

Brian, O. et al, (2012). Cloud Computing. Swiss Academy of Engineering Sciences. Retrieved May 2013 from: http://www.cloud-finder.ch/fileadmin/Dateien/PDF/News/2012-1106_SATW_White_Paper_Cloud_Computing_EN_1_.pdf

Katz, R. Goldstein, P. Yanosky, R. (2009) Cloud Computing in Higher Education. EDUCAUSE. Retrieved April 2013 from: http://net.educause.edu/section_params/conf/CCW10/highered.pdf

Mircea, M. (2010) SOA, BPM and Cloud Computing: Connected for innovation in higher education. Retrieved May 2013 from: http://ieeexplore.ieee.org/xpl/login.jsp?tp=\&arnumber=5657616 


\section{AUTHORS' BIOGRAPHIES}

\section{Support to clouds- Service Activity leader: Andres Steijaert, SURFnet}

Andres Steijaert is the activity leader for the European cloud activity at GÉANT: the pan-European data network dedicated to the research and education community, connecting 50 million users. At SURF, as a member of the SURFnet-taskforce Cloud he initiated and directed the cloud brokerage and vendor management activities. Previously he worked as a program manager on the development of the SURFconext collaboration infrastructure, a middleware framework which interconnects cloud vendors and higher education organizations in the Netherlands. Andres is a frequent speaker, nationally and internationally, on cloud computing.

\section{Cloud strategy - Task leader: Fulvio Galeazzi, GARR}

Fulvio Galeazzi is a technology researcher at GARR and manager of the DECIDE project (https://www.eu-decide.eu), A FP7 project, providing the Neuroscientific and Medical community with a dedicated Grid-based e-Infrastructure, relying on the Pan-European backbone GEANT and the NRENs. He also works on the GARR cloud strategy and the GARRbox cloud storage service. Fulvio has been with GARR since 2010. Previously, he was involved with projects EUMEDGRID-Support and EUChinaGRID (2005-2009) and with the BaBar, ATLAS and Argo experiments (2002-2010). He holds a $\mathrm{PhD}$ in Physics from the University of Padova.

\section{Cloud standards and interoperability - Task leader: Miroslav Ruda, CESNET}

Miroslav Ruda currently works as the head of the grid department at CESNET and is also a researcher at CESNET and Masaryk University. He leads the Czech NGI (NGI_CZ) group in the EGI InSPIRE project and since 2010 he is leading the Czech national grid called MetaCentrum. He is and has been involved in many international projects (e.g. GridLab, CoreGRID, DataGrid, EGEE I, II, III, EGI InSpire). Since 2011, he is leading the joint cloud activity of CESNET and CERIT-SC called MetaCloud and represents CESNET in several cloud-related activities, including EGI FedCloud group.

\section{Cloud brokerage and vendor management - Task leader: Branko Radojevic, CARNet}

Branko Radojevic is a Deputy CEO at CARNet, in charge of the Department of Computer and Data Infrastructure, which is responsible for designing, building and maintaining CARNet's computer and data infrastructure. He received his B.Sc. and M.Sc. degrees in CIS/MIS from the Columbia Southern University (Alabama, US) and it is expected to earn his Ph.D. in Computer Science from University of Zagreb, Faculty of Electrical Engineering and Computing in 2014. Branko is currently leading the national VoIP project and several other key projects in educational field in Croatia. He was involved in the GN2 JRA1 and GN3 JRA1 activities and currently in TERENA TF-STORAGE activities.

\section{Cloud integration - Task leader: Panos Louridas, GRNET}

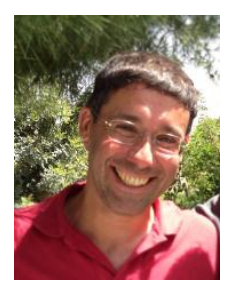

Panos Louridas holds a PhD and an MSc in Software Engineering from the University of Manchester, and a Diploma in Computer Science from the University of Athens. He has been working in GRNET since 2005, focusing on the area of computing infrastructures. He was involved in the EGEE series of projects, serving as NA5 leader and as the country representative. More recently, he is the country representative in the EGI-InSPIRE project. In the last few years he has been heavily involved in the area of cloud computing, and serves as the project manager for the flagship cloud computing project in Greece (budget over 18M euro), which develops and delivers the Okeanos cloud computing platform (https://okeanos.grnet.gr/home/). Dr. Louridas has published widely in all areas of Software Engineering, and is an active researcher in the Athens University of Economics and Business, and also a software practitioner. He is a member of the ACM, the IEEE, Usenix, and the AAAS. 


\section{Product Marketing Leader: Karl Meyer, Dante}

Karl holds a BEng in Mechanical Engineering from the University of Southampton and an MBA from the Open University. He has been working within the Internet industry for nearly 20 years within both Technical and Consulting roles. As Senior Network Consultant at WorldCom his clients included major ISPs, on-line retailers and manufacturers both in the areas of technical design and marketing strategy. His work within the Cloud Computing environment extends back to 1998 with the launch of virtual data centre services within WorldCom and the development of the Messagelabs virtualised virus scanning service with Star Internet.

\section{About GÉANT}

As the pan-European data network dedicated to the research and education community, GÉANT connects 40 million users to the internet. Through its innovative access and authentication services of eduroam and eduGAIN, GÉANT has long experience in the fields of user access services and federated service authentication and delivery.

In GÉANT, European National Research and Education Network organizations collaborate on the cloud and address topics like cloud strategy, standards, interoperability, privacy and security, cloud brokerage and procurement, vendor management and integration. 